Review Article

\title{
The Intricate Link among Gut "Immunological Niche," Microbiota, and Xenobiotics in Intestinal Pathology
}

\author{
Danilo Pagliari, ${ }^{1}$ Giovanni Gambassi, ${ }^{1}$ Ciriaco A. Piccirillo, ${ }^{2,3,4}$ and Rossella Cianci ${ }^{1}$ \\ ${ }^{1}$ Department of Internal Medicine and Medical Sciences, "A. Gemelli Hospital", Catholic University of the Sacred Heart, Rome, Italy \\ ${ }^{2}$ Department of Microbiology and Immunology, McGill University, Montréal, QC, Canada \\ ${ }^{3}$ Program in Infectious Disease and Immunity in Global Health, Research Institute of the McGill University Health Centre, \\ Montréal, QC, Canada \\ ${ }^{4}$ Centre of Excellence in Translational Immunology (CETI), Research Institute of the McGill University Health Centre, \\ Montréal, QC, Canada
}

Correspondence should be addressed to Rossella Cianci; rossellacianci@gmail.com

Received 5 May 2017; Revised 6 July 2017; Accepted 12 July 2017; Published 8 October 2017

Academic Editor: Tânia Silvia Fröde

Copyright (c) 2017 Danilo Pagliari et al. This is an open access article distributed under the Creative Commons Attribution License, which permits unrestricted use, distribution, and reproduction in any medium, provided the original work is properly cited.

Inflammatory bowel diseases (IBDs) are diseases characterized by various degrees of inflammation involving the gastrointestinal tract. Ulcerative colitis and Crohn's disease are characterized by a dysregulated immune response leading to structural gut alterations in genetically predisposed individuals. Diverticular disease is characterized by abnormal immune response to normal gut microbiota. IBDs are linked to a lack of physiological tolerance of the mucosal immune system to resident gut microbiota and pathogens. The disruption of immune tolerance involves inflammatory pathways characterized by an unbalance between the anti-inflammatory regulatory $\mathrm{T}$ cells and the proinflammatory Th1/ Th17 cells. The interaction among $\mathrm{T}$ cell subpopulations and their related cytokines, mediators of inflammation, gut microbiota, and the intestinal mucosa constitute the gut "immunological niche." Several evidences have shown that xenobiotics, such as rifaximin, can positively modulate the inflammatory pathways at the site of gut immunological niche, acting as anti-inflammatory agents. Xenobiotics may interfere with components of the immunological niche, leading to activation of anti-inflammatory pathways and inhibition of several mediators of inflammation. In summary, xenobiotics may reduce disease-related gut mucosal alterations and clinical symptoms. Studying the complex interplay between gut immunological niche and xenobiotics will certainly open new horizons in the knowledge and therapy of intestinal pathologies.

\section{The Role of Mucosal Immunity in the Intestinal Mucosal Barrier}

Human bowel has a sophisticated immune system that protects from pathogen's infections, while maintaining a tolerance to food antigens and nonpathogen bacteria [1]. The mucus layer over the gut epithelium itself contains antimicrobial products and secretory IgA and it is the first defensive component. However, it is the intestinal epithelium with its secretory antibacterial peptides [2] and innate and adaptive immune system cells that regulates gut immunity (Figure 1). Intestinal mucosal immune cells are specifically organized to form a so-called gut-associated lymphoid tissue (GALT), where cells are activated by bacterial antigens. These structural and immunological defense mechanisms of the human gut have been referred to as the "mucosal firewall" [3].

It has been well established that $\mathrm{CD} 4^{+} \mathrm{T}$ cells can differentiate into several subtypes that may have both pro- and anti-inflammatory properties $[4,5]$. Th1, Th2, and Th17 cells generate mucosal inflammation and tissue damage while regulatory $\mathrm{T}$ cells (Tregs), instead, have anti-inflammatory properties and limit mucosal inflammation and promote tissue repair. Thus, $\mathrm{T}$ cell subsets and their related cytokines contribute to the physiological maintenance and the 


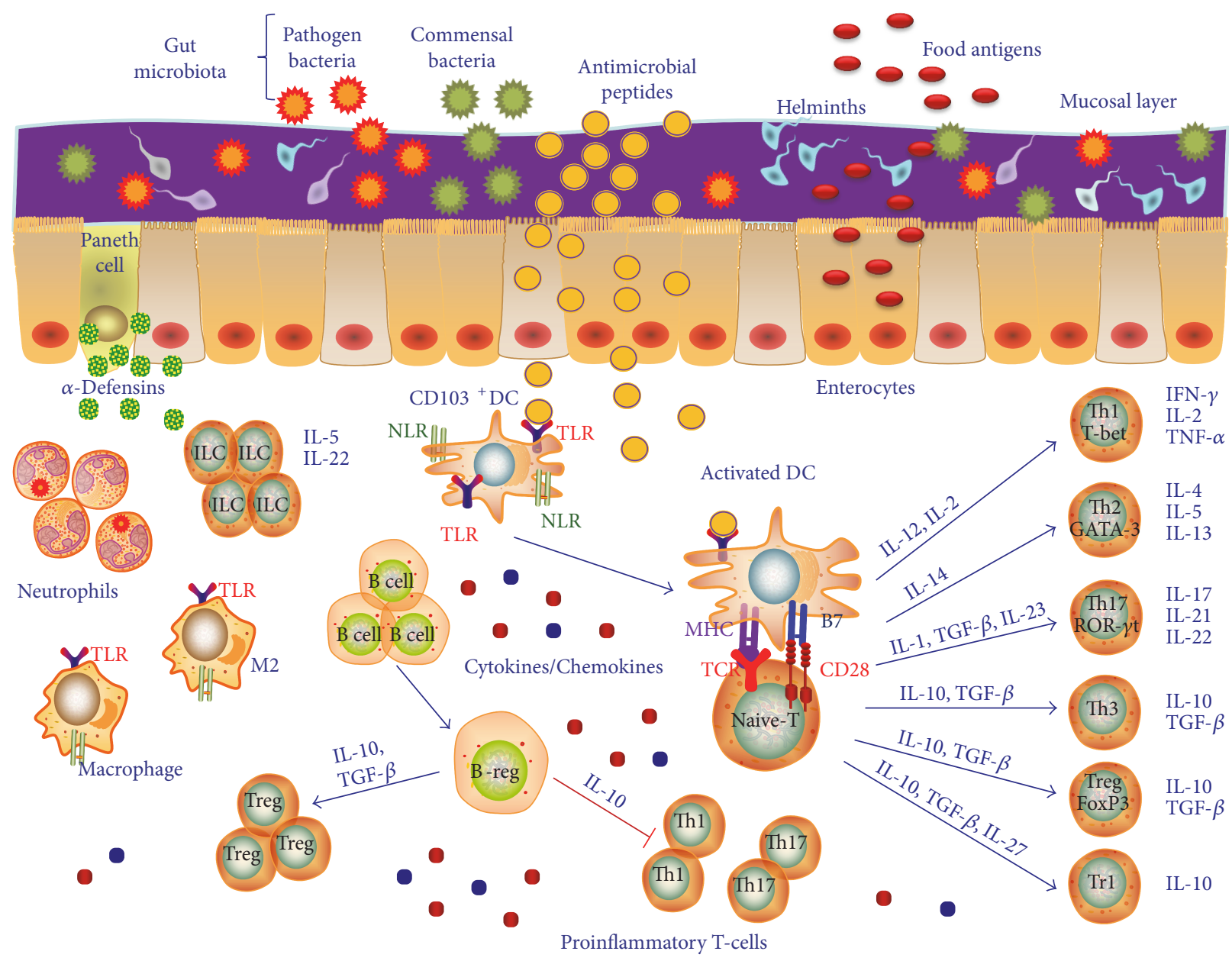

FIgURE 1: The complex interactions in the gut "immunological niche". The human bowel is a sophisticated immune system that protects from pathogen's infections. The intestinal mucosal layer represents a mechanical barrier. The mucus over the gut epithelium contains antimicrobial peptides and it is the first defensive component. However, it is the intestinal epithelium with its secretory antibacterial peptides, innate and adaptive immune system cells, and their related pro- and anti-inflammatory cytokines and chemokines that regulate gut immunity. Intestinal mucosal immune cells are specifically organized to form a so-called gut-associated lymphoid tissue (GALT), where cells are activated by bacterial antigens. These structural and immunological defense mechanisms of the human gut have been referred to as the “immunological niche." TLR: Toll-like receptors; Treg: regulatory T cells; NLR: NOD-like receptors; TCR: T cell receptor; IL: interleukin; GM-CSF: granulocyte-macrophage colony-stimulating factor; DC: dendritic cell; MHC: major histocompatibility complex; PD1: programmed death 1; PD-L1: programmed death-ligand 1.

pathological transformation of intestinal mucosa by constantly modulating the gut homeostasis and inflammation [6]. These T cell subpopulations present in the gut mucosa are associated with a specific cytokine cocktail [5]. Thus, several cytokines and their receptors with pro- and antiinflammatory functions resulted involved in inflammatory diseases of the bowel $[5,7]$, such as IFN $-\gamma$ and IFN $-\gamma$ R1, TNF- $\alpha$, IL-1R1, IL-2 and IL-2RA, IL-4, IL-5, IL-9, IL-12 $\beta$ and IL-12R, IL-13, IL-15, IL-17A, IL-18, IL-21, IL-22, IL-23 and IL-23R, TGF- $\beta$, IL-10, and IL-27 $[8,9]$. The wellknown proinflammatory $\mathrm{T}$ cell subpopulations are Th1 cells that are characterized by the specific production of IFN- $\gamma$ and IL-12 and the counter-regulatory Th2 cells which produce humoral immunity-promoting cytokines like IL-4, IL-5, and IL-13. IL-12, a major product of activated DC, stimulates Th1 differentiation and production of effector cytokines, such as IFN- $\gamma$ and TNF- $\alpha$. As a classic activator of cell-mediated immunity, IFN- $\gamma$ activates macrophages, natural killer (NK) cells, and $\mathrm{CD}^{+} \mathrm{T}$ cells. For Th2 cells, IL-4 acts as the major Th2 differentiation factor and promotes IL-4 and IL-13 expression. In particular, IL-13 is a cytokine with proinflammatory functions, as it participates in the disruption of gut epithelial barrier and in the promotion of mucosal fibrosis via TGF- $\beta 1$ expression [10]. Furthermore, another proinflammatory $\mathrm{T}$ cell subset is constituted by Th 9 cells characterized by the production of IL-9. IL-9 acts impairing gut mucosal healing, barrier function, and epithelial cell proliferation [11].

Th17 cells are key initiators of proinflammatory responses in gut mucosal surfaces. Th17 cells via their production of IL-17A and IL-17F are generally proinflammatory and play an important role in host defense against infection to extracellular pathogens, by recruiting neutrophils and macrophages to infected tissues. Their development depends 
on signals mediated by IL-6 (and downstream activation of STAT3) and TGF- $\beta$, IL-21, and IL-23 and by induction of the lineage-specifying transcription factor, retinoic acidrelated orphan nuclear receptor $(\mathrm{ROR} \gamma \mathrm{T})$ [5]. IL-17A is involved in local chronic inflammation inducing proinflammatory cytokine expression leading to mucosal destruction and altering mucosal healing. Among Th17 cell cytokines, a key role is played by IL-23 that orchestrates the survival and maintenance of the Th17 phenotype and in turn the crosstalk between innate and adaptive immunity in the gut [12]. Interestingly, aberrant expression and activity of the IL-17/IL-23 axis is frequently involved in the pathogenesis of several inflammatory bowel pathologies $[13,14]$. Consistently, studies report that polymorphisms in the il23r gene are associated with inflammatory bowel diseases (IBDs) $[15,16]$. Another proinflammatory cytokine implicated in IBD pathogenesis is IL-21 which is secreted by $\mathrm{T}$ follicular helper (Tfh) cells, and it is implicated in the differentiation of germinal center B cells into high-affinity antibodysecreting plasma cells and memory B cells that ensure sustained immune protection and rapid recall responses against previously encountered foreign antigens [17]. IL-21 can also play important roles in $\mathrm{T}$ cell subset development. Tfh cells differentiate from naïve $\mathrm{CD}^{+}{ }^{+} \mathrm{T}$ helper cell precursors after antigen activation in the presence of IL- 6 and IL- 21 and induction of B cell lymphoma 6 (BcL6) $[4,18]$. It must be noted that while Th17 cells are prominent inducers of chronic inflammatory responses in disease states of the gut, this subset can protect the intestinal mucosa from microbiota and pathogens by its ability to resist infection and promote IgA secretion. Moreover, Th17 cells also produce IL-22 which has important functions in host defense at mucosal surfaces as well as in tissue repair [19]. While it is produced by innate lymphoid cells (ILCs) and Th cell subsets, including Th17 cells, IL-22 acts only on nonhematopoietic stromal cells like epithelial cells and keratinocytes. Although IL-22 is beneficial to the host in many infectious and inflammatory disorders, it can be pathologic due to its proinflammatory properties, which are further enhanced by other proinflammatory cytokines like IL-17 [20].

Several innate-like lymphocyte populations are involved in key homeostatic and pathogenic interactions with gut microbiota. Among these populations, a crucial role is played by ILCs, of which exist 3 subpopulations: ILC1, ILC2, and ILC3 [21]. These cells are part of the innate immune system, their actions are strictly related to the presence of commensal microbiota, and they interact between both the innate and adaptive arms of the immune system [22]. As it relates to the regulation of intestinal immune responses, ILC3 participates in the maintenance of mucosal barrier homeostasis by producing the anti-inflammatory cytokine IL-22, and they stimulate neutrophils and macrophage recruitment and proliferation in the gut, producing IL-17 and granulocyte-macrophage colony-stimulating factor (GM-CSF), respectively. In turn, macrophages may stimulate ILC function by producing IL-1 $\beta$ [23]. On the other hand, ILCs can stimulate T cells by favoring antigen presentation by intramucosal antigenpresenting cells (APCs), such as DCs [22].
The abovementioned cytokines and chemokines are principally related to several $\mathrm{T}$ cell subtypes of the adaptive immunity. Among these molecules, some of them have a bridge action in participating in both innate and adaptive immune response and include cytokines like IFN- $\gamma$, TNF- $\alpha$, IL-1, IL-2, IL-12, and IL-15 [24]. Among these cytokines, a special role is played by IL-15 which plays key roles in the intestinal mucosal barrier [25]. IL-15 is a member of the IL2 family of cytokines whose signaling pathway constitutes a bridge between innate and adaptive immune responses. On one hand, IL-15 regulates the differentiation and activation of proinflammatory Th1 and Th17 cells, and on the other hand, it blocks the activation of the immunosuppressive Tregs. In addition, IL-15 may mediate enterocyte apoptosis [26]. Consistent with these actions, several studies have reported an upregulation of IL-15 in inflamed tissues of patients with bowel pathologies, such as IBDs, and diverticular disease [27]. These data suggest that IL-15 may exert a direct pathogenetic role in these conditions. However, there is also evidence that IL-15 may potentiate the immune response against cancer. For these reasons, IL-15 is still considered both a friend and a foe of human physiology and pathology [28].

An essential role of the immune system is to eradicate pathogens while suppressing the potential for immune pathology. Triggering and maintaining immune tolerance within the intestine represent a unique challenge to the mucosal immune system. A variety of immune-regulatory cell subsets within the T cell, B cell, dendritic cells (DCs), and macrophage (M2 phenotype) compartments, each endowed with unique suppressive functions, are critical for ensuring sustained immune tolerance in the intestinal tissue microenvironment through active inhibition of innate and adaptive immune responses [20].

One of the predominant anti-inflammatory cell types are Tregs, of which there are many subtypes [29] including $\mathrm{CD} 4^{+} \mathrm{CD} 25^{\text {high }}$ Treg cells. These cells are characterized by the expression of the forkhead box P3 (FoxP3), the master-switch, lineage-specifying transcription factor that orchestrates the transcriptional landscape and drives the development and function of this Treg subset. FoxP ${ }^{+}$Tregs are essential mediators of immune tolerance by modulating innate and adaptive immune responses to self and nonself antigens [30]. Developmental, homeostatic, or functional deficits in these cells can provoke autoimmune disease and all the while augment responses to pathogens, tumors, or allergens [29]. Foxp $3^{+}$Tregs also have a positive role in limiting tissue inflammation, maintaining immune tolerance, and promoting mucosal healing in the gut [31]. In fact, studies have shown that their number is inversely correlated with the clinical course and severity of IBDs [6]. Moreover, the development of Tregs is strictly linked to the presence of commensal gut microbiota [32]. In fact, evidence in experimental mouse models showed that, in the absence of gut microbiota, the number of Tregs resulted significantly reduced and it was subsequently restored to normal proportions after gut recolonization with flora $[33,34]$.

DCs play an important role in activating immune responses but also in the induction of tolerance to microbial 
and dietary antigens [35]. DCs are environmental sentinels scanning for various innate and danger signals and poised in tissue to influence the immune activation or suppression decision. To this end, they are present in the mesenteric lymph nodes, in the gut lamina propria, and in Peyer's patches and participate in the control of intestinal inflammation [36]. Normally, DCs, particularly the tolerogenic $\mathrm{CD} 103^{+} \mathrm{DC}$ subset, are recruited in the gut during inflammatory conditions and in turn efficiently act stimulating the differentiation of Foxp $3^{+}$Tregs [37]. Overall, Foxp $3^{+}$Tregs, in concertation with other immunoregulatory cell types, including T regulatory 1 ( $\operatorname{Tr} 1)$ cells, T helper 3 (Th3) cells, regulatory $\mathrm{B}$ cells (Bregs), $\mathrm{CD}_{103}{ }^{+} \mathrm{DCs}$, and $\mathrm{M} 2$ cells, are instrumental in establishing a global context of immune tolerance to a spectrum of potentially pathogenic microorganisms in the gut flora [20].

\section{The Gut Microbiota and Mucosal Immunity Crosstalk}

Gut microbiota is the collection of all microbial populations that reside in the gastrointestinal tract. It can weigh up to a total of $1 \mathrm{~kg}$ and contains tens of trillions of microorganisms, a 100 times more genes than the host, and includes at least 1000 different bacterial species. It is increasingly recognized that gut microbiota plays a pivotal role in the gastrointestinal tract homeostasis [38]. A strong reciprocal interaction between gut microbiota and host immunity has been proven as the former has coevolved in a symbiotic relationship with mucosal immunity. These commensal bacteria are called "keystone species," and overall, they can be considered a "superorganism" that is an integral part of the human gastrointestinal tract [39].

The human intestine has a large surface area that constitutes an entrance door for the antigens that we introduce with the food. In addition, the human gut is covered with many bacterial communities, some of which may be dangerous for the host. Hence, the principal role of the intestinal immune system is to protect the host from pathogens preventing infections. To this end, in mucosal immune cells, several surface receptors are endowed that mediate the interaction with microbial antigens. Among these pattern recognition receptors (PRRs), the most important are the Toll-like receptors (TLRs) and the NOD-like receptors (NLRs) [40].

In physiological homeostasis, there is a perfect balance between microbial load and the immune response generated against it [41]. The immune system correctly functions to ensure tolerance to food antigens and defense against microbial infections. A homeostatic role is played by TLR signaling. Commensal microbiota also participates to immune tolerance by promoting the differentiation of antiinflammatory Tregs [42]. Conversely, in a disease state or during an infection, the normal gut homeostasis is lost with an excess of tissue inflammation. In this altered environment, TLRs, activated by pathogens, lose their homeostatic role and promote the activation and development of an inflammatory response, contributing to acute and chronic intestinal inflammatory states [43].
Host and microbial metabolisms are also key modulators of innate and adaptive immune responses in mucosal environments. While both occur simultaneously, the two are profoundly interdependent: while the host depends on the microbiome for a spectrum of digestive and metabolic enzymes, the microbiota, particularly in the gut, produces a wide array of metabolites from endogenous compounds produced by microbes and the host [44], but primarily from the anaerobic fermentation of dietary components in the colon [45]. The epithelial cell layer that constitutes the host microbe mucosal interface permits microbial-derived metabolic products to access and interact with host cells and, in turn, shape downstream inflammatory and immune responses. One salient example of such metabolite is shortchain fatty acids (SCFAs), like propionic acid and butyric acid, which are produced by colonic microbial fermentation of undigested or partially digested dietary fibres. They signal in host cells via G protein-coupled receptors (e.g., GPR41 and GPR43) on the surface of epithelial and immune cells, having a range of effects on host immune functions [46]. The effects of SCFAs include inhibition of histone deacetylase activity and altered gene expression in host cells and augmented epithelial barrier function which promotes gut homeostasis via several mechanisms: (1) enhanced mucus production by intestinal goblet cells, (2) inhibition of NF- $\kappa \mathrm{B},(3)$ activation of inflammasomes and IL-18 production, (4) increased B cell secretion of secretory $\operatorname{IgA}$, (5) diminished maturation of DCs, and (6) increased number and function of colonic Foxp ${ }^{+}$Tregs. SCFAs are not restricted to the gut and can also find their way to other organs, such as the lungs, where they directly or indirectly act on local APCs to modulate inflammatory responses that are associated with airway disease (allergic or infectious) [46-49].

\section{Microbiota and Host Immune System Interactions in Inflammatory Bowel Diseases}

IBDs, such as Crohn's disease (CD) and ulcerative colitis (UC), are chronic and multifactorial pathologies of the gastrointestinal tract. In these bowel diseases, there is an imbalance between proinflammatory and anti-inflammatory responses, but their full pathogenetic mechanisms are still incompletely understood [50]. It is known that in genetically predisposed individuals, an inappropriate immune response against luminal agents is activated with an abnormal production of cytokines and other mediators of inflammation [9]. The genetic factors involved in the pathogenesis of IBDs include genes encoding proteins of immunity involved in environmental sensing of microbial-derived products and signals.

In particular, nucleotide-binding oligomerization domain-containing protein 2 (NOD2) is an autophagyrelated gene that activates an immune reaction against components of the bacteria cell wall, including peptidoglycans, in both Gram-positive and Gram-negative bacteria such as in the case of Shigella and Listeria [51]. NOD2 is expressed on the cell surface of various epithelial and innate immune cells, such as neutrophils, DCs, stromal cells, macrophages, and others [52]. NOD2 has several homeostatic 
functions like intracellular bacterial sensing, inducing the expression of several antibacterial peptides, such as $\alpha$-defensin, and participating in the immune tolerance mechanisms by the suppression of the TLR axis [53]. Under physiological conditions, when the equilibrium between pro- and antiinflammatory factors is maintained in the gut, NOD2 expression levels are very low [51]. On the other hand, in pathological situations, when proinflammatory factors are predominant over the anti-inflammatory ones, NOD2 level expressions are increased, particularly due to the overexpression of the proinflammatory cytokine TNF- $\alpha$ [51]. The activation of NOD2 by antimicrobial peptides determines the initiation of a signaling cascade which is responsible for the production of several proinflammatory cytokines and chemokines involving the activation of NF- $\kappa \mathrm{B}$ [52]. The breakdown of the physiological equilibrium between immunity and microbiota determines alterations in the NOD2 functions [54]. The bidirectional function of NOD2 is linked to the inflammatory levels present on the gut surface. Interestingly, NOD2 polymorphisms are associated with an increased risk for the development of $\mathrm{CD}$, and aberrant NOD2 activity may alter the functions of both epithelial and innate immunity cells. Primarily, NOD2 in the epithelial compartment is able to selectively reduce the levels of alphadefensins in Paneth cells in the small bowel $[55,56]$. NOD2 activation is also linked to disturbance of hematopoietic cells and DC activity [57]. Moreover, NOD2 polymorphisms are related to a reduction of nuclear factor kappa-light-chainenhancer of activated B cells (NF- $\kappa \mathrm{B})$ in response to bacterial peptidoglycan [58]. Finally, other genetic factors involved in the pathogenesis of IBDs include the signal transducer and activator of transcription 3 (STAT3), and IL-23 receptor (IL-23R) [25].

Both humoral and cell mediated immunity are involved in the pathogenesis of IBDs [59]. IBDs are also characterized by an influence of environmental factors. In fact, IBDs pathogenesis is influenced by the TLR axis activated by both commensal and pathogenetic bacteria, leading to the promotion of inflammatory pathways responsible for tissue damage [25]. Likewise, IBDs are characterized by a profound modification of the gut microbiota. Typically, pathogens grow and proliferate to suppress the physiological flora and generate a dysbiotic state. The consequent intestinal barrier dysfunction determines the translocation of pathogens in the lamina propria. Human GALT is directly affected in at least two distinct ways. First, the outgrowth of opportunistic classes of bacteria drives increased inflammation [60]. In this scenario, TLRs mediate the activation of the proinflammatory transcription factor NF- $\kappa \mathrm{B}$ with the consequent production of proinflammatory cytokines and chemokines [61]. Secondly, the loss of benign fermenting bacteria that produce "keystone" metabolites results in reduced immunoregulation [3]. In particular, studies report that IBDs present specific gut microbiota alterations, characterized by a reduction in bacterial diversity and an increase in bacterial instability [13]. There is an increase of Bacteroidetes and Proteobacteria in both $\mathrm{CD}$ and UC $[62,63]$. CD is further characterized by an increase in some pathogens of the Enterobacteriaceae family [64], such as Salmonella, Shigella, Escherichia, invasive
Fusobacteria, and Actinobacteria [62]. Likewise, both CD and UC present a reduced number of Firmicutes, Lachnospiraceae, and Ruminococcaceae [65]. Finally, in CD, fewer Bifidobacteria have been reported [66].

Considering the immunopathogenesis of IBDs, it is well known that $\mathrm{CD}$ is related to a Th1 and Th17 immune profile, while UC is related to a Th2 response [67]. Then, both pathologies are characterized by the unbalance between proinflammatory $\mathrm{T}$ cell subsets and anti-inflammatory Tregs [59]. In fact, both in CD and UC, Tregs and their related antiinflammatory cytokines resulted as decreased. Several literature data have reported a strong interaction among mucosal immunity, TLRs, and gut microbiota. These interactions may regulate gut physiology, immune tolerance to external dietary antigens, protection from external infections, regulation of gut microbial populations, and the commensal/pathogen ratio. A special focus has been placed on TLR2 and TLR4 signaling. In fact, their signals promote Treg proliferation and survival [68]. Then, other evidences supported the fact that such bacteria may directly influence the development of Th17 cell differentiation. In this way, Bacteroides fragilis promotes Treg differentiation and IL-10 and TGFBeta production and inhibits Th17 cell differentiation [69].

\section{Pharmacological Modulation of the Immunological Niche}

These data suggest that the intestinal mucosa holds a complex immune-functional set that is central in the regulation of physiological homeostasis. For this reason, the gut mucosa may be considered an "immunological niche," that is, a definite immune-functional region, that is, constituted by $\mathrm{T}$ cell subpopulations and their related anti- and proinflammatory cytokines, several mediators of inflammation, and gut microbiota $[26,70]$. Perturbations and disruption of the immunological niche are critical steps in the pathogenetic pathways contributing to the development of inflammatory bowel pathologies. However, it is likely that we may also use the concept of immunological niche to explain the mechanisms of several other "inflammatory" diseases. An immediate therapeutic strategy would be to use agents that can modulate the immunological niche reducing inflammation and rebalancing gut immunity [71]. While there is no such definitely proven agent, there is a growing interest in the possible role of xenobiotics, especially rifaximin [72].

\section{The Role of Xenobiotics in the Interactions with the Immunological Niche: The Case of Rifaximin}

Xenobiotics are chemical molecules that are not normally produced by humans. They may interfere with host metabolism and produce effects of modification of the host physiology and pathology. Among xenobiotics, rifaximin seems to be of special interest [73]. Rifaximin is a semisynthetic agent based on rifamycin with a broad-action spectrum of antibiotic activity against both $\mathrm{Gram}^{+}$and $\mathrm{Gram}^{-}$bacteria. Rifaximin 


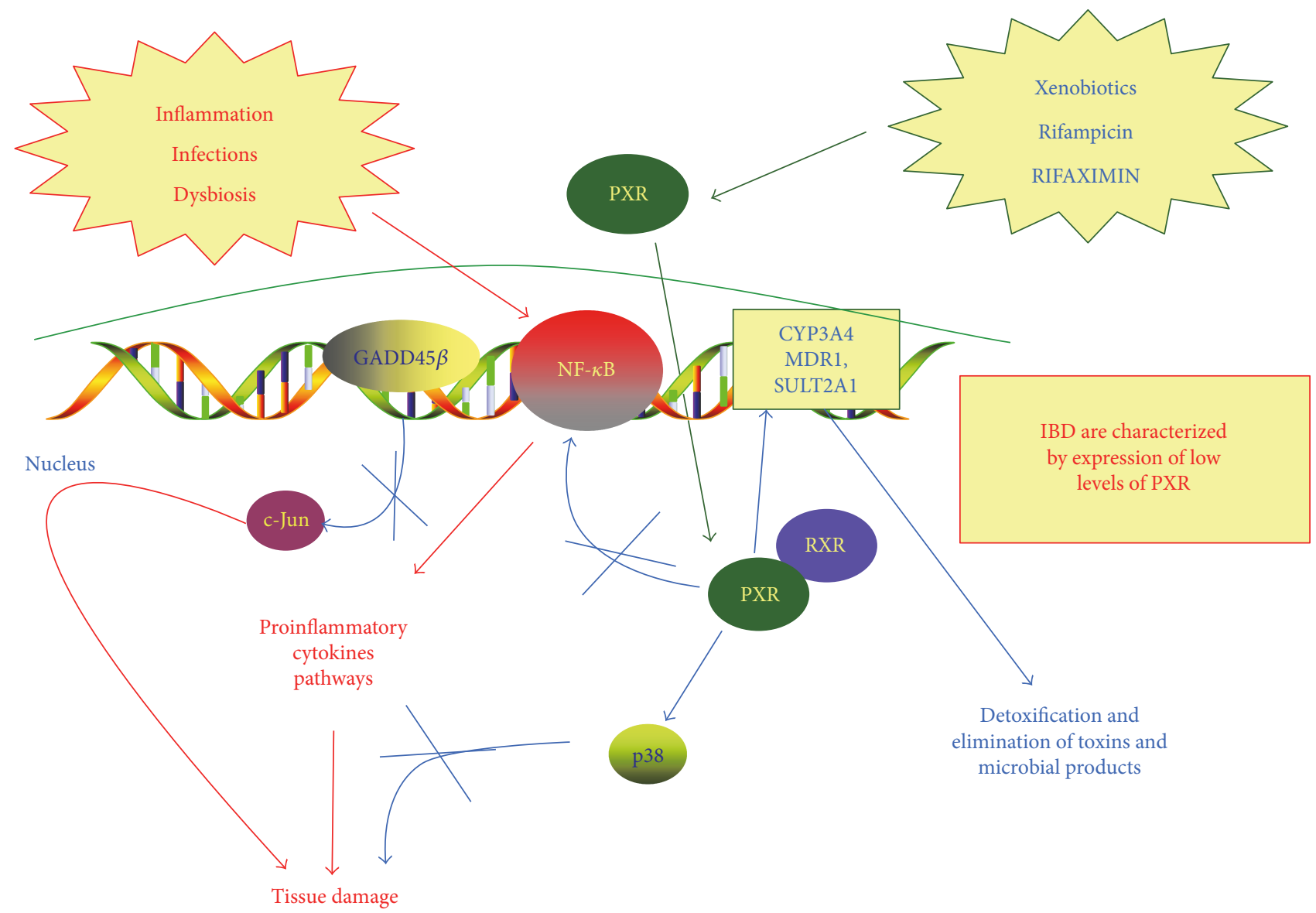

FIGURE 2: The role of rifaximin in modulating gut inflammation via the PXR/NF- $\kappa$ B pathway. Rifaximin is an effective agonist of the nuclear receptor PXR. PXR, greatly expressed in liver and intestinal mucosa, acts as a driver of detoxification processes and contributes to intestinal cell survival during exposure to several xenobiotics. After being activated by its ligands, PXR translocates into the nucleus where it binds its receptor and then regulates DNA transcription. The anti-inflammatory effects of rifaximin may not only be linked to the reduction of ileal bacteria load but it may also have an indirect action on inflammation. In fact, rifaximin, being an effective agonist of PXR, may regulate the inflammatory process. In particular, rifaximin, activating PXR, can inhibit NF- $\kappa$ B activity and the consequent transcription of several proinflammatory cytokines, such as TNF-alpha and IL- $1 \beta$. The activation of PXR then upregulates p38 MAP kinase signal cascade via GADD $45 \beta$ upregulation. All these mechanisms are linked to the prevention of tissue damage and to favor gut mucosal healing.

has poor oral bioavaibility; thus, it acts locally in the gastrointestinal tract having only few systemic effects [74].

Differently from common antibiotics, rifaximin may modify gut microbiota toward a relative abundance of certain species of protective bacteria. Several data have confirmed that rifaximin is able to increase the proliferation and growth of the protective Lactobacilli while inhibiting that of several pathobionts, including Clostridia and Firmicutes, with negative effects $[75,76]$. These changes in microbiota composition may contribute to the anti-inflammatory effects of rifaximin on the intestinal mucosa [77]. Indeed, Lactobacilli are able to downregulate mucosal inflammation, improving the function of intestinal barrier, and restoring the normal mucosal permeability [78].

The anti-inflammatory effects of rifaximin may not only be linked to the reduction of ileal bacteria load but it may also have an indirect action on inflammation. In fact, rifaximin is an effective agonist of the nuclear receptor PXR [79]. PXR, greatly expressed in liver and intestinal mucosa, acts as a driver of detoxification processes and contributes to intestinal cell survival during exposure to several xenobiotics. After being activated by its ligands, PXR translocates into the nucleus where it binds its receptor and then regulates DNA transcription (Figure 2). PXR can inhibit NF- $\kappa \mathrm{B}$ activity and the consequent transcription of several proinflammatory cytokines, such as TNF-alpha and IL-1beta [73, 80]. In 2010, Cheng et al. [81] demonstrated that rifaximin reduces the expression of the NF- $\kappa \mathrm{B}$-related proinflammatory genes activating PXR function. In contrast, rifaximin is not able to modify the expression of these genes in PXR-null transgenic mice. For this reason, PXR is considered an antiinflammatory molecular factor. In support of this, it has been proven that IBDs are characterized by the expression of low levels of PXR. Thus, rifaximin activation of PXR function may have positive anti-inflammatory properties. Finally, rifaximin may limit inflammation-mediated damage activating the p38 MAP kinase that is directly able to promote tissue repair [82]. 


\section{The Role of Rifaximin in Inflammatory Bowel Diseases}

It is well known that gut microbiota plays an important role in the development of IBDs [83]. Thus, the modulation of the gut microbiota has been put as focus of several clinical and research areas [84]. The manipulation of intestinal bacteria can be achieved by several modalities that involved xenobiotics, such as prebiotics, probiotics, and antibiotics, and fecal transplants $[85,86]$.

Prebiotics are fermentable substances participating in the modulation of gut flora. Prebiotics have several actions on the gut mucosa, such as the improvement of IL-10 DCs and TLR2 and TLR4 cells. Then, they exert positive modulation of gut microbiota populations improving the growth of beneficial resident bacteria as a consequence of the manipulation of the luminal substrate composition. Moreover, prebiotics improve the intestinal barrier and regulate the mucosal immune system [87].

On the other hand, probiotics are live microorganisms that, administered in therapeutic doses, confer a health advantage on the host. Probiotics present several positive actions on the gut mucosa. In fact, probiotics restore the microbial balance, protect the host against pathogens, and modify gut-associated lymphoid tissue and the mucosal immune system. A synergic action between prebiotics and probiotics seems to be associated with a reduction of the concentration of pathogenetic metabolites and dangerous microflora [85].

In particular, several antibiotics may modulate the course of IBDs by reducing pathological bacteria, such as Escherichia coli, Bacteroides fragilis, and other gram-negative Enterobacteriaceae present in the gut lumen, and by altering the composition of gut microbiota to favor beneficial bacteria [83]. Literature data demonstrated that antibiotics may be effectively used in the treatment of IBDs, due to their action in reducing bacterial overgrowth, resolving septic systemic complications and local infections, such as abscesses and fistulas [88]. Importantly, it has been shown that antibiotics may be used even to maintain IBD remission [89]. Among xenobiotics, rifaximin seems to influence remission in both CD and UC [90].

In 2005, Shafran and Johnson performed a clinical study on active CD patients. Rifaximin was administered at a dose of $200 \mathrm{mg}$ twice daily for 16 weeks, and it induced a high clinical remission (59\% cases) [91]. Then, in a further study, Shafran and Burgunder performed a retrospective analysis of CD patients receiving adjunctive therapy with rifaximin (mean dose $600 \mathrm{mg}$ daily for 16 weeks) showing clinical remission in a high amount of cases (70\%) [92]. In 2012, Prantera et al. performed a clinical trial comparing the twice-daily rifaximin administration of $400 \mathrm{mg}, 800 \mathrm{mg}$, and $1200 \mathrm{mg}$ versus placebo. This trial demonstrated that the administration of rifaximin $800 \mathrm{mg}$ twice daily for 3 months was able to induce clinical remission of moderately active CD [93]. Surprisingly, the administration of rifaximin $1200 \mathrm{mg}$ and $400 \mathrm{mg}$ twice daily for 3 months had no significant higher induction of clinical CD remission versus placebo [93]. The treatment resulted safe and well tolerated by all patients. Furthermore, a similar trial on active, moderate $\mathrm{CD}$ reported significantly higher rates of remission after 12 weeks of treatment among patients receiving rifaximin $800 \mathrm{mg}$ twice daily versus placebo. Thus, rifaximin may be used as an adjunct to standard therapy, although the authors did not address the surprisingly high clinical remission rates observed in both the rifaximin and placebo groups [94].

Differently from CD, data on the efficacy of rifaximin in UC are anecdotal [95]. In particular, in 1999, a clinical trial with rifaximin in unresponsive-to-steroids UC patients was conducted. This trial demonstrated that $400 \mathrm{mg}$ rifaximin twice daily for 10 days significantly reduced clinical symptoms and mucosal healing in those patients [96]. In 2006, Guslandi et al. conducted a small clinical trial on $30 \mathrm{UC}$ patients with steroid intolerance. Rifaximin $400 \mathrm{mg}$ twice daily was added for 4 weeks to the mesalamine $2.4 \mathrm{~g}$ daily treatment, and clinical remission was obtain in a large amount of cases $[97,98]$. In addition, it has been evaluated that the combined treatment with Saccharomyces boulardii $500 \mathrm{mg}$ daily plus rifaximin $400 \mathrm{mg}$ daily did obtain clinical remission in all mesalamine-resistant UC patients [99].

Recently, a clinical trial has been conducted with a fully humanized anti-IL-17A monoclonal antibody in CD patients [100]. However, this trial failed in this disease but it had satisfactory results in psoriasis [101]. In fact, literature data confirmed that the overexpression in the IL-17/IL-23 axis related to the increased level in Th17 cells constitutes a favorable prognostic factor in the pathogenesis of IBDs $[6,13]$.

Other innovative therapies for IBDs have been developed using humanized antibodies against the cytokines overexpressed in these diseases. To date, several anti-cytokine antibodies are approved for IBD therapy, including anti-TNFs (infliximab, etanercept, adalimumab, golimumab, certolizumab, etc.), anti-IL-17 (secukinumab, brodalumab), anti-IL12/23 p40 (ustekinumab), and IL-23 p19 (tildrakizumab) [9].

\section{The Role of Rifaximin in Diverticular Disease}

A better understanding of the potential role of rifaximin is derived from a series of studies conducted by our group in patients with diverticular disease. In 2009, we demonstrated that in diverticular disease, there are several modifications in $\mathrm{T}$ cell subpopulations, both in peripheral blood and in colonic mucosa. In particular, these patients have an increased tissue recruitment of $\mathrm{CD} 103^{+}$lymphocytes [102]. These cells are characteristic of the intestinal homing, because they typically move from the peripheral blood to the gut mucosa [70].

It has been now well established that in patients with diverticular disease, rifaximin is able to ameliorate clinical symptoms reducing bacterial overgrowth and related mucosal chronic inflammation. Indeed, gut $\mathrm{CD}_{103^{+}}$lymphocytes are reduced after 2 months of rifaximin treatment. This is the first demonstration that rifaximin has the ability to not only modify gut microbiota and inhibit tissue inflammation but may also directly modulate $T$ cell circulation and mucosal immunity. In 2014, we demonstrated that the number of TLR2 and TLR4 lymphocytes both in peripheral blood and in sigmoid mucosa is significantly altered in diverticular 
patients relative to controls [103]. An increased number of TLR2 and TLR4 cells in the peripheral blood of diverticular patients indicates an increase in activated circulating $\mathrm{T}$ cells. Moreover, after placebo, we demonstrated that the number of TLR2 and TLR4 lymphocytes increased. This evidence indicates that TLRs are indirect markers of bacterial overgrowth. These data reveal that rifaximin may act limiting bacterial overgrowth and then reducing the related TLR activation. TLRs mediate the activation of both innate and adaptive immune response and may also activate the proinflammatory transcriptional factor $\mathrm{NF}-\kappa \mathrm{B}[43]$. NF- $\kappa \mathrm{B}$ has a key role in the development of immune response against pathogenic bacteria. In fact, $\mathrm{NF}-\kappa \mathrm{B}$ is associated with a transcription and secretion of a Th1 proinflammatory cytokine pattern [43]. In this way, these data demonstrate the effective role of rifaximin in modulating local and systemic TLR expression and T cell circulation and further confirm the well-established anti-inflammatory properties of this drug in addition to its antibacterial action [72]. Therefore, considering its multiple activities, rifaximin could be redefined as a "eubiotic" agent acting as a gut microenvironment modulator (Table 1).

\section{Conclusions}

Inflammatory bowel pathologies are a heterogeneous group of diseases characterized by various degrees of inflammation involving the gut mucosa. Several mediators of inflammation are involved in their pathogenesis, such as T cell subpopulations and their related pro- and anti-inflammatory cytokines, TLRs, and the pathogen/commensal ratio. A strong interaction among these factors has been well evaluated. In fact, on the one hand, gut microbiota may strictly modulate mucosal immunity, and on the other hand, mucosal immunity may influence the composition of gut microbiota. This complex interaction between gut microbiota and mucosal immunity is also mediated by other several factors that participate in the inflammatory pathways.

Inflammatory bowel pathologies are shared by the disruption of the physiological homeostasis present in the gut mucosa. First, the alteration in the pathogen/commensal ratio precipitates in the pathological condition of dysbiosis. During dysbiosis, the normal physiology of the gut mucosa is altered and there is bacterial translocation from the lumen to systemic circulation due to the leaky gut condition. In this pathological scenario, there is a dysregulation in the cytokine production, with the proliferation of proinflammatory $\mathrm{T}$ cell subsets and inhibition of anti-inflammatory ones. All these complex interactions among $\mathrm{T}$ cell subpopulations, gut microbiota, and the mediators of inflammation occur in the anatomical subset of the gut mucosa, which we have defined as "immunological niche."

Several evidences have shown that xenobiotics may positively modulate the gut immunological niche. Most specifically, xenobiotics may interfere with components of the immunological niche, leading to an activation of antiinflammatory pathways, and inhibition of several inflammatory mediators. As a result, xenobiotics may reduce diseaserelated gut mucosal alterations and clinical symptoms.
TABLE 1: The beneficial effects of the interaction between gut microbiota and immunological niche: the role of rifaximin as a "eubiotic" agent.

Rifaximin may act on both innate and adaptive immune cells and has a role on both mucosal and systemic immunity. Thus, it may have 3 levels of action

Positively selecting commensal gut microbial communities:

(i) Increasing the proliferation and growth of the protective Lactobacilli

(1) Gut microbiot

(ii) Inhibiting the proliferation of several pathobionts, including Clostridia and Firmicutes

Inhibiting the PXR-induced transcription of

(2) Inflammation NF- $\kappa$ B proinflammatory-related genes, such as TNF-alpha and IL-1beta

(i) Reducing TLR activation

\begin{tabular}{ll} 
(3) Mucosal & (ii) Interfering with T cell circulation and gut \\
and systemic & homing of CD103 ${ }^{+}$lymphocytes and \\
immunity & inhibiting proinflammatory T cells, such as \\
& Th1 and Th17 cells \\
\hline
\end{tabular}

In summary, while further research is warranted, the complex interplay between gut immunological niche and xenobiotics has the potential to open new horizons in our knowledge of inflammatory bowel pathologies and their treatment.

\section{Conflicts of Interest}

The authors declare that there is no conflict of interest regarding the publication of this paper.

\section{References}

[1] K. J. Maloy and F. Powrie, "Intestinal homeostasis and its breakdown in inflammatory bowel disease," Nature, vol. 474, no. 7351, pp. 298-306, 2011.

[2] R. Okumura and K. Takeda, "Roles of intestinal epithelial cells in the maintenance of gut homeostasis," Experimental \& Molecular Medicine, vol. 49, no. 5, article e338, 2017.

[3] Y. Belkaid and T. W. Hand, "Role of the microbiota in immunity and inflammation," Cell, vol. 157, no. 1, pp. 121141, 2014.

[4] S. Nakayamada, H. Takahashi, Y. Kanno, and J. J. O'Shea, "Helper T cell diversity and plasticity," Current Opinion in Immunology, vol. 24, no. 3, pp. 297-302, 2012.

[5] S. Bhaumik and R. Basu, "Cellular and molecular dynamics of Th17 differentiation and its developmental plasticity in the intestinal immune response," Frontiers in Immunology, vol. 8, p. 254, 2017.

[6] F. Pandolfi, R. Cianci, D. Pagliari, R. Landolfi, and G. Cammarota, "Cellular mediators of inflammation: Tregs and $\mathrm{T}_{\mathrm{H}} 17$ cells in gastrointestinal diseases," Mediators of Inflammation, vol. 2009, Article ID 132028, 11 pages, 2009.

[7] K. H. Katsanos and K. A. Papadakis, "Inflammatory bowel disease: updates on molecular targets for biologics," Gut and Liver, vol. 11, no. 4, pp. 455-463, 2017. 
[8] L. Jostins, S. Ripke, R. K. Weersma et al., "Host-microbe interactions have shaped the genetic architecture of inflammatory bowel disease," Nature, vol. 491, no. 7422, pp. 119124, 2012.

[9] M. L. Chen and M. S. Sundrud, "Cytokine networks and Tcell subsets in inflammatory bowel diseases," Inflammatory Bowel Diseases, vol. 22, no. 5, pp. 1157-1167, 2016.

[10] K. Gyires, E. V. Toth, and S. Z. Zadori, "Gut inflammation: current update on pathophysiology, molecular mechanism and pharmacological treatment modalities," Current Pharmaceutical Design, vol. 20, no. 7, pp. 1063-1081, 2014.

[11] B. Weigmann and M. F. Neurath, "Th9 cells in inflammatory bowel diseases," Seminars in Immunopathology, vol. 39, no. 1, pp. 89-95, 2017.

[12] C. S. Catana, I. Berindan Neagoe, V. Cozma, C. Magdaş, F. Tăbăran, and D. L. Dumitrașcu, "Contribution of the IL17/IL-23 axis to the pathogenesis of inflammatory bowel disease," World Journal of Gastroenterology, vol. 21, no. 19, pp. 5823-5830, 2015.

[13] G. Cammarota, G. Ianiro, R. Cianci, S. Bibbò, A. Gasbarrini, and D. Currò, "The involvement of gut microbiota in inflammatory bowel disease pathogenesis: potential for therapy," Pharmacology \& Therapeutics, vol. 149, pp. 191-212, 2015.

[14] A. Luo, S. T. Leach, R. Barres, L. B. Hesson, M. C. Grimm, and D. Simar, "The microbiota and epigenetic regulation of $\mathrm{T}$ helper 17/regulatory T cells: in search of a balanced immune system," Frontiers in Immunology, vol. 8, p. 417, 2017.

[15] Z. K. Lu, Z. R. Chen, J. Y. Zhu, Y. Xu, and X. Hua, “Analysis of the association of single nucleotide polymorphisms of interleukin-23 receptor (IL-23R) and inflammatory bowel disease in a Chinese Han cohort," Oncotarget, vol. 7, no. 42, pp. 67851-67856, 2016.

[16] L. Song, R. Zhou, S. Huang et al., "High intestinal and systemic levels of interleukin-23/T-helper 17 pathway in Chinese patients with inflammatory bowel disease," Mediators of Inflammation, vol. 2013, Article ID 425915, 10 pages, 2013.

[17] E. Troncone, I. Marafini, F. Pallone, and G. Monteleone, "Th17 cytokines in inflammatory bowel diseases: discerning the good from the bad," International Reviews of Immunology, vol. 32, no. 5-6, pp. 526-533, 2013.

[18] W. K. Suh, "Life of T follicular helper cells," Molecules and Cells, vol. 38, no. 3, pp. 195-201, 2015.

[19] L. Brockmann, A. D. Giannou, N. Gagliani, and S. Huber, "Regulation of $\mathrm{T}_{\mathrm{H}} 17$ cells and associated cytokines in wound healing, tissue regeneration, and carcinogenesis," International Journal of Molecular Sciences, vol. 18, no. 5, 2017.

[20] M. Sun, C. He, Y. Cong, and Z. Liu, "Regulatory immune cells in regulation of intestinal inflammatory response to microbiota," Mucosal Immunology, vol. 8, no. 5, pp. 969-978, 2015.

[21] M. Gury-BenAri, C. A. Thaiss, N. Serafini et al., "The spectrum and regulatory landscape of intestinal innate lymphoid cells are shaped by the microbiome," Cell, vol. 166, no. 5, pp. 1231.e13-1246.e13, 2016.

[22] C. A. Thaiss, N. Zmora, M. Levy, and E. Elinav, "The microbiome and innate immunity," Nature, vol. 535, no. 7610, pp. 65-74, 2016.

[23] A. I. Lim, T. Verrier, C. A. Vosshenrich, and J. P. Di Santo, "Developmental options and functional plasticity of innate lymphoid cells," Current Opinion in Immunology, vol. 44, pp. 61-68, 2017.
[24] S. Zundler and M. F. Neurath, "Pathogenic T cell subsets in allergic and chronic inflammatory bowel disorders," Immunological Reviews, vol. 278, no. 1, pp. 263-276, 2017.

[25] A. Geremia, P. Biancheri, P. Allan, G. R. Corazza, and A. Di Sabatino, "Innate and adaptive immunity in inflammatory bowel disease," Autoimmunity Reviews, vol. 13, no. 1, pp. 310, 2014.

[26] D. Pagliari, R. Cianci, S. Frosali et al., "The role of IL-15 in gastrointestinal diseases: a bridge between innate and adaptive immune response," Cytokine \& Growth Factor Reviews, vol. 24, no. 5, pp. 455-466, 2013.

[27] M. J. Tosiek, L. Fiette, S. El Daker, G. Eberl, and A. A. Freitas, "IL-15-dependent balance between Foxp3 and ROR $\gamma \mathrm{t}$ expression impacts inflammatory bowel disease," Nature Communications, vol. 7, article 10888, 2016.

[28] D. Pagliari, S. Frosali, R. Landolfi, and R. Cianci, "The role of IL-15 in human cancer: friend or foe?," International Trends in Immunity, vol. 1, no. 2, pp. 35-42, 2013.

[29] N. Kamada, S. U. Seo, G. Y. Chen, and G. Núñez, "Role of the gut microbiota in immunity and inflammatory disease," Nature Reviews Immunology, vol. 13, no. 5, pp. 321-335, 2013.

[30] D. Bauche and J. C. Marie, "Transforming growth factor $\beta$ : a master regulator of the gut microbiota and immune cell interactions," Clinical \& Translational Immunology, vol. 6, no. 4, article e136, 2017.

[31] C. Pedros, F. Duguet, A. Saoudi, and M. Chabod, "Disrupted regulatory $\mathrm{T}$ cell homeostasis in inflammatory bowel diseases," World Journal of Gastroenterology, vol. 22, no. 3, pp. 974-995, 2016.

[32] S. Omenetti and T. T. Pizarro, "The Treg/Th17 axis: a dynamic balance regulated by the gut microbiome," Frontiers in Immunology, vol. 6, p. 639, 2015.

[33] N. Kamada, Y. G. Kim, H. P. Sham et al., "Regulated virulence controls the ability of a pathogen to compete with the gut microbiota," Science, vol. 336, no. 6086, pp. 1325-1329, 2012.

[34] J. D. Lord, "Promises and paradoxes of regulatory T cells in inflammatory bowel disease," World Journal of Gastroenterology, vol. 21, no. 40, pp. 11236-11245, 2015.

[35] J. Farache, E. Zigmond, G. Shakhar, and S. Jung, "Contributions of dendritic cells and macrophages to intestinal homeostasis and immune defense," Immunology \& Cell Biology, vol. 91, no. 3, pp. 232-239, 2013.

[36] S. C. Knight, "Dendritic cell-T-cell circuitry in health and changes in inflammatory bowel disease and its treatment," Digestive Diseases, vol. 34, no. 1-2, pp. 51-57, 2016.

[37] S. Rutella and F. Locatelli, "Intestinal dendritic cells in the pathogenesis of inflammatory bowel disease," World Journal of Gastroenterology, vol. 17, no. 33, pp. 37613775, 2011.

[38] J. R. Marchesi, D. H. Adams, F. Fava et al., "The gut microbiota and host health: a new clinical frontier," Gut, vol. 65, no. 2, pp. 330-339, 2016.

[39] G. Hajishengallis, R. P. Darveau, and M. A. Curtis, "The keystone-pathogen hypothesis," Nature Reviews Microbiology, vol. 10, no. 10, pp. 717-725, 2012.

[40] B. K. Davis, C. Philipson, R. Hontecillas, K. Eden, J. Bassaganya-Riera, and I. C. Allen, "Emerging significance of NLRs in inflammatory bowel disease," Inflammatory Bowel Diseases, vol. 20, no. 12, pp. 2412-2432, 2014. 
[41] D. Pagliari, C. A. Piccirillo, A. Larbi, and R. Cianci, “The interactions between innate immunity and microbiota in gastrointestinal diseases," Journal of Immunology Research, vol. 2015, Article ID 898297, 3 pages, 2015.

[42] A. Ueno, H. Jijon, R. Chan et al., "Increased prevalence of circulating novel IL-17 secreting Foxp3 expressing $\mathrm{CD}^{+} \mathrm{T}$ cells and defective suppressive function of circulating Foxp $3^{+}$regulatory cells support plasticity between Th17 and regulatory $\mathrm{T}$ cells in inflammatory bowel disease patients," Inflammatory Bowel Diseases, vol. 19, no. 12, pp. 2522-2534, 2013.

[43] S. Frosali, D. Pagliari, G. Gambassi, R. Landolfi, F. Pandolfi, and R. Cianci, "How the intricate interaction among Tolllike receptors, microbiota, and intestinal immunity can influence gastrointestinal pathology," Journal of Immunology Research, vol. 2015, Article ID 489821, 12 pages, 2015.

[44] L. Lin and J. Zhang, "Role of intestinal microbiota and metabolites on gut homeostasis and human diseases," BMC Immunology, vol. 18, no. 1, p. 2, 2017.

[45] R. Havenaar, "Intestinal health functions of colonic microbial metabolites: a review," Beneficial Microbes, vol. 2, no. 2, pp. 103-114, 2011.

[46] L. Macia, J. Tan, A. T. Vieira et al., "Metabolite-sensing receptors GPR43 and GPR109A facilitate dietary fibre-induced gut homeostasis through regulation of the inflammasome," Nature Communications, vol. 6, article 6734, 2015.

[47] R. Correa-Oliveira, J. L. Fachi, A. Vieira, F. T. Sato, and M. A. Vinolo, "Regulation of immune cell function by short-chain fatty acids," Clinical \& Translational Immunology, vol. 5, no. 4, article e73, 2016.

[48] J. Tan, C. McKenzie, P. J. Vuillermin et al., "Dietary fiber and bacterial SCFA enhance oral tolerance and protect against food allergy through diverse cellular pathways," Cell Reports, vol. 15, no. 12, pp. 2809-2824, 2016.

[49] G. Goverse, R. Molenaar, L. Macia et al., "Diet-derived short chain fatty acids stimulate intestinal epithelial cells to induce mucosal tolerogenic dendritic cells," Journal of Immunology, vol. 198, no. 5, pp. 2172-2181, 2017.

[50] Q. Guan and J. Zhang, "Recent advances: the imbalance of cytokines in the pathogenesis of inflammatory bowel disease," Mediators of Inflammation, vol. 2017, Article ID 4810258, 8 pages, 2017.

[51] S. Coutermarsh-Ott, K. Eden, and I. C. Allen, "Beyond the inflammasome: regulatory NOD-like receptor modulation of the host immune response following virus exposure," The Journal of General Virology, vol. 97, no. 4, pp. 825838, 2016.

[52] D. J. Philpott, M. T. Sorbara, S. J. Robertson, K. Croitoru, and S. E. Girardin, "NOD proteins: regulators of inflammation in health and disease," Nature Reviews Immunology, vol. 14, no. 1, pp. 9-23, 2014.

[53] T. Iida, K. Onodera, and H. Nakase, "Role of autophagy in the pathogenesis of inflammatory bowel disease," World Journal of Gastroenterology, vol. 23, no. 11, pp. 1944-1953, 2017.

[54] D. Corridoni, K. O. Arseneau, M. G. Cifone, and F. Cominelli, "The dual role of nod-like receptors in mucosal innate immunity and chronic intestinal inflammation," Frontiers in Immunology, vol. 5, p. 317, 2014.

[55] J. P. Hugot, M. Chamaillard, H. Zouali et al., "Association of NOD2 leucine-rich repeat variants with susceptibility to Crohn's disease," Nature, vol. 411, no. 6837, pp. 599-603, 2001.
[56] P. P. Elia, Y. F. Tolentino, C. Bernardazzi, and H. S. de Souza, "The role of innate immunity receptors in the pathogenesis of inflammatory bowel disease," Mediators of Inflammation, vol. 2015, Article ID 936193, 10 pages, 2015.

[57] R. Cooney, J. Baker, O. Brain et al., "NOD2 stimulation induces autophagy in dendritic cells influencing bacterial handling and antigen presentation," Nature Medicine, vol. 16, no. 1, pp. 90-97, 2010.

[58] J. Wehkamp, J. Harder, M. Weichenthal et al., "NOD2 (CARD15) mutations in Crohn's disease are associated with diminished mucosal $\alpha$-defensin expression," Gut, vol. 53, no. 11, pp. 1658-1664, 2004.

[59] J. H. Park, L. Peyrin-Biroulet, M. Eisenhut, and J. I. Shin, "IBD immunopathogenesis: a comprehensive review of inflammatory molecules," Autoimmunity Reviews, vol. 16, no. 4, pp. 416-426, 2017.

[60] D. Sheehan and F. Shanahan, "The gut microbiota in inflammatory bowel disease," Gastroenterology Clinics of North America, vol. 46, no. 1, pp. 143-154, 2017.

[61] A. Achek, D. Yesudhas, and S. Choi, "Toll-like receptors: promising therapeutic targets for inflammatory diseases," Archives of Pharmacal Research, vol. 39, no. 8, pp. 10321049, 2016.

[62] A. W. Walker, J. D. Sanderson, C. Churcher et al., "Highthroughput clone library analysis of the mucosa-associated microbiota reveals dysbiosis and differences between inflamed and non-inflamed regions of the intestine in inflammatory bowel disease," BMC Microbiology, vol. 11, p. 7, 2011.

[63] D. N. Frank, A. L. S. Amand, R. A. Feldman, E. C. Boedeker, N. Harpaz, and N. R. Pace, "Molecular-phylogenetic characterization of microbial community imbalances in human inflammatory bowel diseases," Proceedings of the National Academy of Sciences of the United States of America, vol. 104, no. 34, pp. 13780-13785, 2007.

[64] R. B. Sartor, "Microbial influences in inflammatory bowel diseases," Gastroenterology, vol. 134, no. 2, pp. 577-594, 2008.

[65] C. R. Hedin, N. E. McCarthy, P. Louis et al., "Altered intestinal microbiota and blood $\mathrm{T}$ cell phenotype are shared by patients with Crohn's disease and their unaffected siblings," Gut, vol. 63, no. 10, pp. 1578-1586, 2014.

[66] A. Andoh, H. Kuzuoka, T. Tsujikawa et al., "Multicenter analysis of fecal microbiota profiles in Japanese patients with Crohn's disease," Journal of Gastroenterology, vol. 47, no. 12, pp. 1298-1307, 2012.

[67] X. R. Xu, C. Q. Liu, B. S. Feng, and Z. J. Liu, “Dysregulation of mucosal immune response in pathogenesis of inflammatory bowel disease," World Journal of Gastroenterology, vol. 20, no. 12, pp. 3255-3264, 2014.

[68] S. Wang, L. M. Charbonnier, M. Noval Rivas et al., "MyD88 adaptor-dependent microbial sensing by regulatory $\mathrm{T}$ cells promotes mucosal tolerance and enforces commensalism," Immunity, vol. 43, no. 2, pp. 289-303, 2015.

[69] J. L. Round, S. M. Lee, J. Li et al., "The Toll-like receptor 2 pathway establishes colonization by a commensal of the human microbiota," Science, vol. 332, no. 6032, pp. 974977, 2011.

[70] R. Cianci, D. Pagliari, V. Pietroni, R. Landolfi, and F. Pandolfi, "Tissue infiltrating lymphocytes: the role of cytokines in their growth and differentiation," Journal of Biological Regulators \& Homeostatic Agents, vol. 24, no. 3, pp. 239-249, 2010. 
[71] M. F. Neurath, "Current and emerging therapeutic targets for IBD," Nature Reviews Gastroenterology \& Hepatology, vol. 14, no. 5, pp. 269-278, 2017.

[72] R. B. Sartor, "Review article: the potential mechanisms of action of rifaximin in the management of inflammatory bowel diseases," Alimentary Pharmacology \& Therapeutics, vol. 43, Supplement 1, pp. 27-36, 2016.

[73] A. Mencarelli, M. Migliorati, M. Barbanti et al., "Pregnane-Xreceptor mediates the anti-inflammatory activities of rifaximin on detoxification pathways in intestinal epithelial cells," Biochemical Pharmacology, vol. 80, no. 11, pp. 1700-1707, 2010.

[74] S. A. Hirota, "Understanding the molecular mechanisms of rifaximin in the treatment of gastrointestinal disorders - a focus on the modulation of host tissue function," MiniReviews in Medicinal Chemistry, vol. 16, no. 3, pp. 206-217, 2015.

[75] A. Acosta, M. Camilleri, A. Shin et al., "Effects of rifaximin on transit, permeability, fecal microbiome, and organic acid excretion in irritable bowel syndrome," Clinical and Translational Gastroenterology, vol. 7, article e173, 2016.

[76] S. Soldi, S. Vasileiadis, F. Uggeri et al., "Modulation of the gut microbiota composition by rifaximin in non-constipated irritable bowel syndrome patients: a molecular approach," Clinical and Experimental Gastroenterology, vol. 8, pp. 309325, 2015.

[77] F. R. Ponziani, F. Scaldaferri, V. Petito et al., "The role of antibiotics in gut microbiota modulation: the eubiotic effects of rifaximin," Digestive Diseases, vol. 34, no. 3, pp. 269-278, 2016.

[78] L. R. Lopetuso, V. Petito, F. Scaldaferri, and A. Gasbarrini, "Gut microbiota modulation and mucosal immunity: focus on rifaximin," Mini-Reviews in Medicinal Chemistry, vol. 16, no. 3, pp. 179-185, 2015.

[79] G. Esposito, S. Gigli, L. Seguella et al., "Rifaximin, a nonabsorbable antibiotic, inhibits the release of pro-angiogenic mediators in colon cancer cells through a pregnane $\mathrm{X}$ receptor-dependent pathway," International Journal of Oncology, vol. 49, no. 2, pp. 639-645, 2016.

[80] A. Garg, A. Zhao, S. L. Erickson et al., "Pregnane X receptor activation attenuates inflammation-associated intestinal epithelial barrier dysfunction by inhibiting cytokineinduced myosin light-chain kinase expression and c-Jun N-terminal kinase 1/2 activation," The Journal of Pharmacology and Experimental Therapeutics, vol. 359, no. 1, pp. 91-101, 2016.

[81] J. Cheng, Y. M. Shah, X. Ma et al., "Therapeutic role of rifaximin in inflammatory bowel disease: clinical implication of human pregnane X receptor activation," The Journal of Pharmacology and Experimental Therapeutics, vol. 335, no. 1, pp. 32-41, 2010.

[82] J. Terc, A. Hansen, L. Alston, and S. A. Hirota, "Pregnane X receptor agonists enhance intestinal epithelial wound healing and repair of the intestinal barrier following the induction of experimental colitis," European Journal of Pharmaceutical Sciences, vol. 55, pp. 12-19, 2014.

[83] H. F. Hammer, "Gut microbiota and inflammatory bowel disease," Digestive Diseases, vol. 29, no. 6, pp. 550-553, 2011.

[84] H. Zatorski and J. Fichna, "What is the future of the gut microbiota-related treatment? Toward modulation of microbiota in preventive and therapeutic medicine," Frontiers in Medicine, vol. 1, p. 19, 2014.
[85] M. Ganji-Arjenaki and M. Rafieian-Kopaei, "Probiotics are a good choice in remission of inflammatory bowel diseases: a meta analysis and systematic review," Journal of Cellular Physiology, 2017.

[86] G. Cammarota, G. Ianiro, H. Tilg et al., "European consensus conference on faecal microbiota transplantation in clinical practice," Gut, vol. 66, no. 4, pp. 569-580, 2017.

[87] K. A. Verbeke, L. Boesmans, and E. Boets, "Modulating the microbiota in inflammatory bowel diseases: prebiotics, probiotics or faecal transplantation?," The Proceedings of the Nutrition Society, vol. 73, no. 4, pp. 490-497, 2014.

[88] O. Nitzan, M. Elias, A. Peretz, and W. Saliba, "Role of antibiotics for treatment of inflammatory bowel disease," World Journal of Gastroenterology, vol. 22, no. 3, pp. 1078-1087, 2016.

[89] A. Hviid, H. Svanstrom, and M. Frisch, "Antibiotic use and inflammatory bowel diseases in childhood," Gut, vol. 60, no. 1, pp. 49-54, 2011.

[90] L. Biancone, V. Annese, S. Ardizzone et al., "Safety of treatments for inflammatory bowel disease: clinical practice guidelines of the Italian Group for the Study of Inflammatory Bowel Disease (IG-IBD)," Digestive and Liver Disease, vol. 49, no. 4, pp. 338-358, 2017.

[91] I.Shafran and L. K. Johnson, "An open-label evaluation of rifaximin in the treatment of active Crohn's disease," Current Medical Research and Opinion, vol. 21, no. 8, pp. 1165-1169, 2005.

[92] I. Shafran and P. Burgunder, "Adjunctive antibiotic therapy with rifaximin may help reduce Crohn's disease activity," Digestive Diseases and Sciences, vol. 55, no. 4, pp. 1079-1084, 2010.

[93] C. Prantera, H. Lochs, M. Grimaldi et al., "Rifaximinextended intestinal release induces remission in patients with moderately active Crohn's disease," Gastroenterology, vol. 142, no. 3, pp. 473-481.e4, 2012.

[94] A. O. Jigaranu, O. Nedelciuc, A. Blaj et al., "Is rifaximin effective in maintaining remission in Crohn's disease?," Digestive Diseases, vol. 32, no. 4, pp. 378-383, 2014.

[95] M. Guslandi, "Rifaximin in the treatment of inflammatory bowel disease," World Journal of Gastroenterology, vol. 17, no. 42, pp. 4643-4646, 2011.

[96] P. Gionchetti, F. Rizzello, A. Ferrieri et al., "Rifaximin in patients with moderate or severe ulcerative colitis refractory to steroid-treatment: a double-blind, placebo-controlled trial," Digestive Diseases and Sciences, vol. 44, no. 6, pp. 1220-1221, 1999.

[97] M. Guslandi, M. C. Petrone, and P. A. Testoni, "Rifaximin for active ulcerative colitis," Inflammatory Bowel Diseases, vol. 12, no. 4, p. 335, 2006.

[98] M. Guslandi, "Rifaximin for inflammatory bowel disease," Digestive Diseases and Sciences, vol. 55, no. 6, article 1805, 2010.

[99] M. Guslandi, "Saccharomyces boulardii plus rifaximin in mesalamine-intolerant ulcerative colitis," Journal of Clinical Gastroenterology, vol. 44, no. 5, p. 385, 2010.

[100] W. Hueber, B. E. Sands, S. Lewitzky et al., "Secukinumab, a human anti-IL-17A monoclonal antibody, for moderate to severe Crohn's disease: unexpected results of a randomised, double-blind placebo-controlled trial," Gut, vol. 61, no. 12, pp. 1693-1700, 2012.

[101] R. G. Langley, B. E. Elewski, M. Lebwohl et al., "Secukinumab in plaque psoriasis - results of two phase 3 trials," The New England Journal of Medicine, vol. 371, no. 4, pp. 326-338, 2014. 
[102] R. Cianci, F. Iacopini, L. Petruzziello, G. Cammarota, F. Pandolfi, and G. Costamagna, "Involvement of central immunity in uncomplicated diverticular disease," Scandinavian Journal of Gastroenterology, vol. 44, no. 1, pp. 108-115, 2009.

[103] R. Cianci, S. Frosali, D. Pagliari et al., "Uncomplicated diverticular disease: innate and adaptive immunity in human gut mucosa before and after rifaximin," Journal of Immunology Research, vol. 2014, Article ID 696812, 11 pages, 2014. 


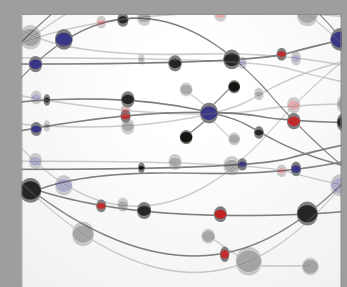

The Scientific World Journal
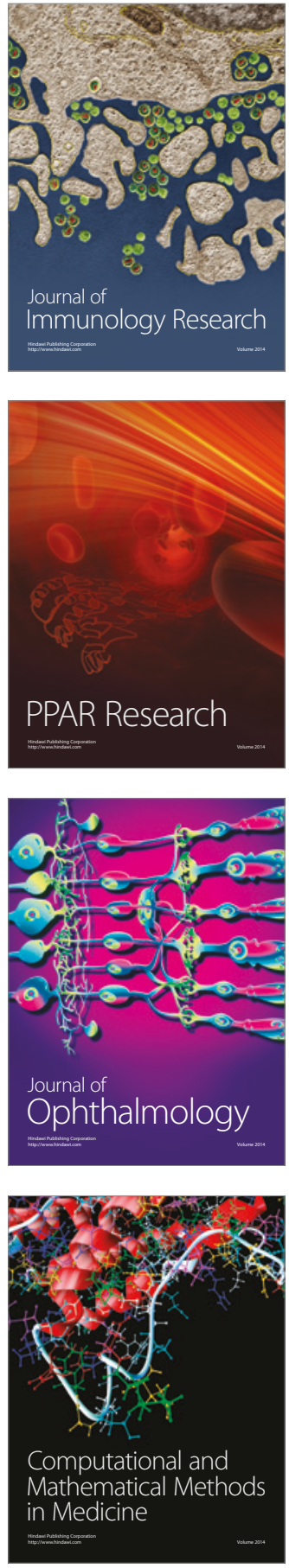

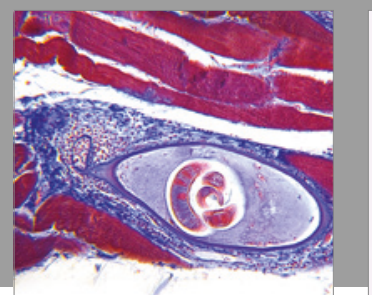

Gastroenterology Research and Practice
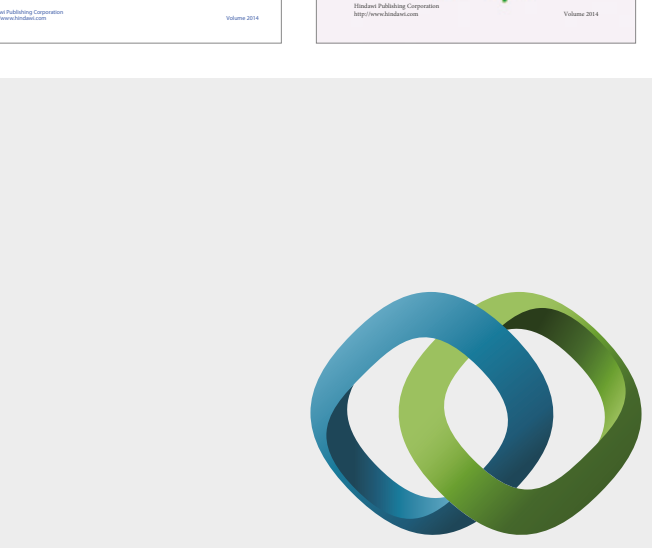

\section{Hindawi}

Submit your manuscripts at

https://www.hindawi.com
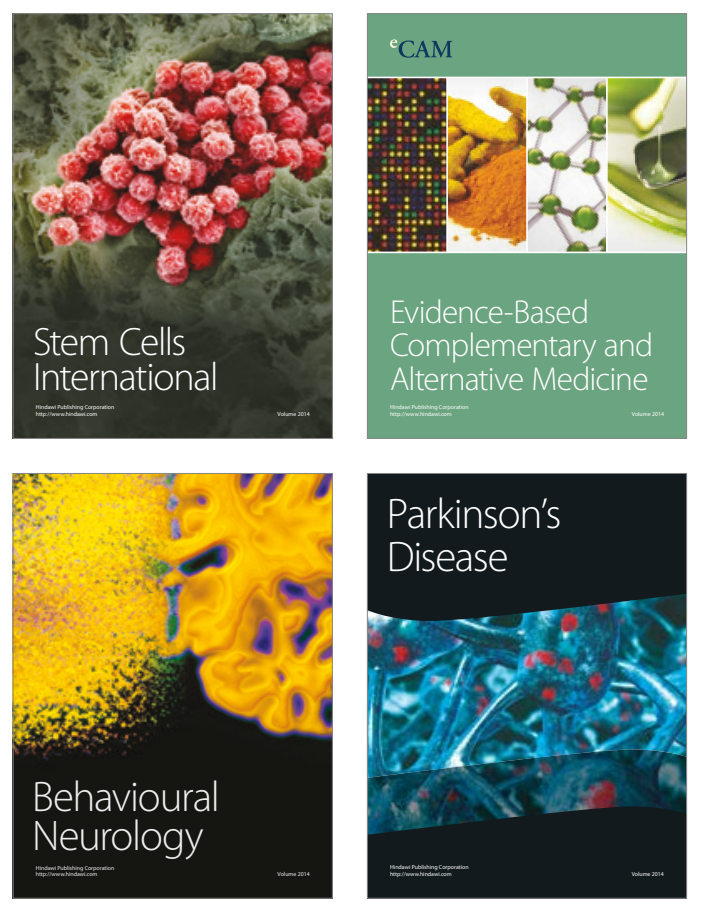
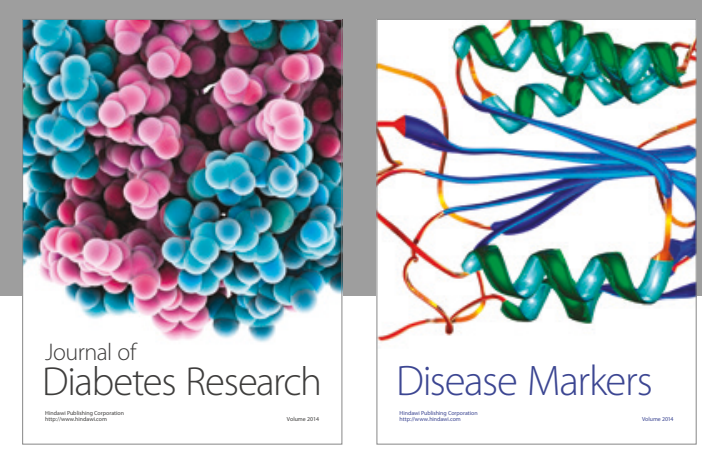

Disease Markers
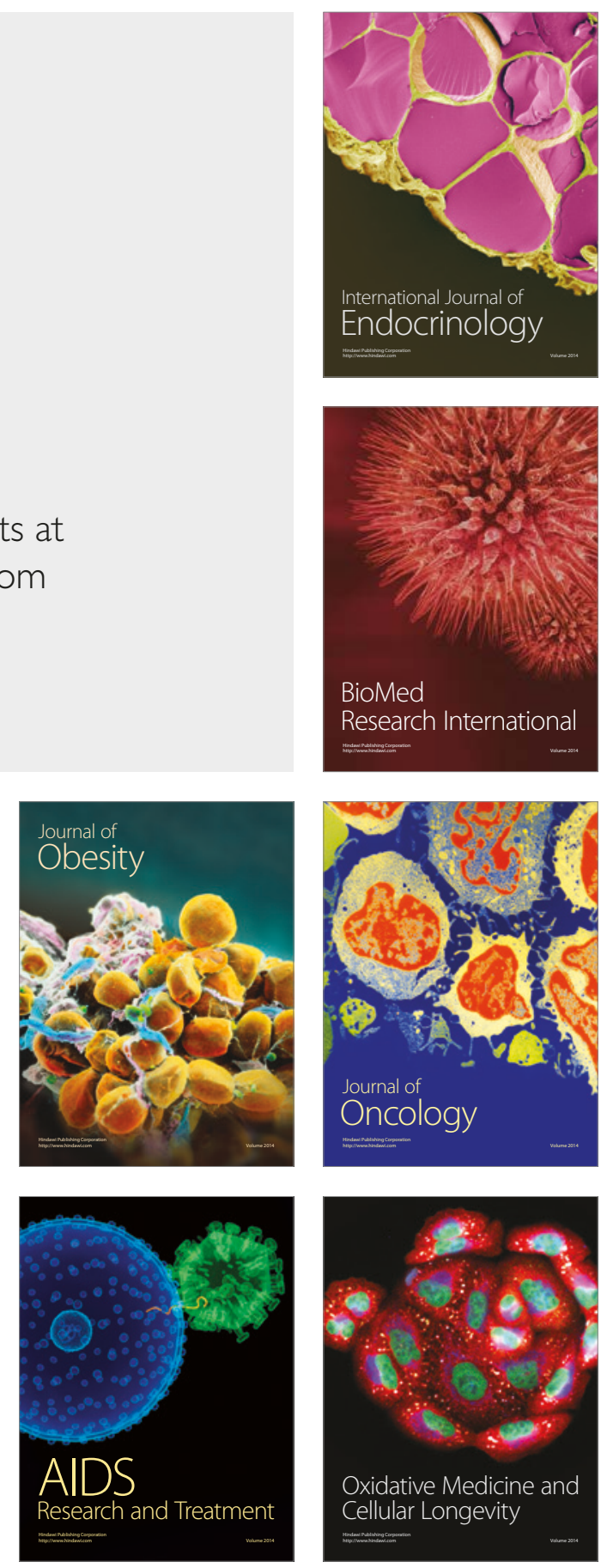